\title{
Pharmacokinetics of Thiamin after Oral Administration of Thiamin Tetrahydrofurfuryl Disulfide to Humans
}

\author{
Nobuyuki KiTAMORI and Yoshinori ITOKAwA ${ }^{1}$ \\ Pharmaceutical Development Laboratories, Takeda Chemical \\ Industries, Ltd., Osaka 532, Japan \\ ${ }^{1}$ Department of Hygiene, Faculty of Medicine, Kyoto University, \\ Sakyo-ku, Kyoto 606, Japan \\ (Received February 5, 1993)
}

\begin{abstract}
Summary We performed a pharmacokinetic analysis of the blood thiamin profile after oral administration of thiamin tetrahydrofurfuryl disulfide (TTFD) to healthy adults. To distinguish between thiamin derived from TTFD ingestion and that from previous dietary intake, the baseline thiamin level was subtracted from the apparent blood vitamin levels measured after administration. Following administration of $100 \mathrm{mg}$ of TTFD, the peak blood thiamin level was almost 10 times the baseline level and the blood thiamin profile could be simulated by a twocompartment model to obtain reasonable pharmacokinetic parameters. When the blood thiamin profile for a 10-mg dose of TTFD was estimated using scaled-down pharmacokinetic parameters derived at the $100-\mathrm{mg}$ dose level, a reasonable fit for the raw data obtained at 10-mg dose was obtained. Therefore, the parameters derived from the data at a dose of $100 \mathrm{mg}$ appeared to be reliable. Since even $180 \mathrm{mg}$ of TTFD is completely absorbed and the absorption ratio is independent of the dose, it can be concluded that gastrointestinal absorption of TTFD is good within the dose range.

Key Words pharmacokinetics, simulation, area under the curve, thiamin, thiamin tetrahydrofurfuryl disulfide (TTFD), blood thiamin level, humans
\end{abstract}

The water-soluble thiamins are absorbed by a saturable, specialized absorption process, and their intestinal absorption is dose-dependent and very poor (1). Consequently, little pharmacokinetic evaluation of thiamin has been performed.

Thiamin tetrahydrofurfuryl disulfide (TTFD) is a thiamin derivative which is readily absorbed from the gastrointestinal tract. Oral administration of TTFD causes a significant increase in the blood thiamin level which is equivalent to that achieved by the intravenous administration of water-soluble thiamins (2). During the absorption of TTFD from the gastrointestinal tract, its S-S bond is broken to 
generate thiamin (3). Therefore, a pharmacokinetic evaluation of the thiamin level after the administration of TTFD should be possible. We have already reported on several studies in which TTFD was administered to healthy male adults (4-6). In the present study, we used the results of these previous investigations for pharmacokinetic analysis.

Another reason for the lack of pharmacokinetic investigations on vitamins may be the difficulty of discriminating between the vitamin derived from the administration of a vitamin preparation and that derived from previous dietary intake. In general, the best method for evaluating the profile of vitamin in blood or plasma is considered to be by using a labeled compound. However, the pharmacokinetic evaluation of blood vitamin level should be based on the intact form of the vitamin alone, while the total radioactivity level may include the metabolites of a vitamin. Thus, even when a labeled compound is used, it is still difficult to evaluate the pharmacokinetics of a vitamin unless its intact form is analyzed separately.

As a simple substitute for the use of labeled compounds, we have proposed that the baseline level of a vitamin can be subtracted from the apparent blood level observed after the administration of an unlabled vitamin preparation (7).

\section{METHODS}

1. Source of the blood thiamin data. Our previous human studies involved the single administration of tablets containing $10 \mathrm{mg}$ of TTFD (equivalent to 6.65 mg of thiamin) (4), the repeated administration of multivitamin tablets containing $10 \mathrm{mg}$ of TTFD for 7 days (4), the single administration of tablets containing 100 $\mathrm{mg}$ of TTFD (5), and the single administration of $100 \mathrm{mg}$ of TTFD powder (6). Informed consent was obtained from each subject at these studies. The mean values $(n=6)$ in these studies were used for the simulation. The experimental methods were described in the previous papers.

2. Pharmacokinetic analysis of blood thiamin data. Simulation of the blood thiamin profile was performed using the program designed by Yamaoka and Tanigawa (8). The parameters of the model equation were selected to obtain the best fit to the data using the least-squares method.

We found that a two-compartment model with first-order absorption could describe the blood thiamin profile with reasonable parameters. When simulation of the blood thiamin profile following oral administration was performed using a model with a lag time before absorption, the results fitted the observed data better.

The two-compartment model with first-order absorption that we used is shown in Fig. 1.

The equation describing the model was as follows:

$$
C_{\mathrm{p}}=A \mathrm{e}^{-\alpha\left(t-t_{0}\right)}+B \mathrm{e}^{-\beta\left(t-t_{0}\right)}-(A+B) \mathrm{e}^{-K_{\mathrm{a}}\left(t-t_{0}\right)},
$$

where 


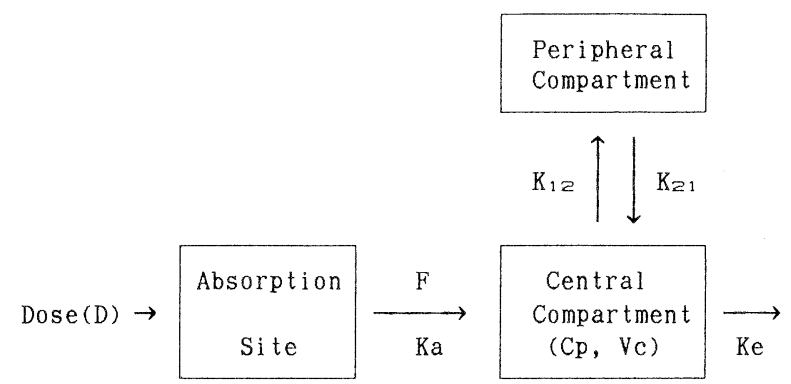

Fig. 1. A two-compartment model with first-order absorption. $K_{12}, K_{21}$ : first-order distribution rate constants; $K_{\mathrm{a}}$ : first-order absorption rate constant; $K_{\mathrm{e}}$ : first-order elimination rate constant; $V_{\mathrm{c}}$ : volume of central compartment; $F$ : fraction of dose absorbed; $C_{\mathrm{p}}$ : vitamin level in central compartment at time $t ; t_{0}$ : lag time.

$$
\begin{aligned}
& \alpha=1 / 2\left[\left(K_{12}+K_{21}+K_{\mathrm{e}}\right)+\left\{\left(K_{12}+K_{21}+K_{\mathrm{e}}\right)^{2}-4 K_{21} K_{\mathrm{e}}\right\}^{1 / 2}\right], \\
& \beta=1 / 2\left[\left(K_{12}+K_{21}+K_{\mathrm{e}}\right)-\left\{\left(K_{12}+K_{21}+K_{\mathrm{e}}\right)^{2}-4 K_{21} K_{\mathrm{e}}\right\}^{1 / 2}\right],
\end{aligned}
$$

and

$$
\begin{aligned}
& A=\frac{K_{\mathrm{a}} F D\left(K_{21}-\alpha\right)}{V_{\mathrm{c}}\left(K_{\mathrm{a}}-\alpha\right)(\beta-\alpha)}, \\
& B=\frac{K_{\mathrm{a}} F D\left(K_{21}-\beta\right)}{V_{\mathrm{c}}\left(K_{\mathrm{a}}-\beta\right)(\alpha-\beta)} .
\end{aligned}
$$

The meaning of each symbol appears in the legend of Fig. 1.

The observed blood thiamin data were fitted to Eq. (1) by the least-squares method to determine the constants $A, B, \alpha, \beta$, and $K_{\mathrm{a}}$. Using these constants, the pharmacokinetic parameters were calculated as follows:

$$
\begin{aligned}
& K_{21}=\frac{A \beta K_{\mathrm{a}}+B \alpha K_{\mathrm{a}}-(A+B) \alpha \beta}{A\left(K_{\mathrm{a}}-\alpha\right)+B\left(K_{\mathrm{a}}-\beta\right)}, \\
& K_{\mathrm{e}}=\frac{\alpha \beta}{K_{21}}, \\
& K_{12}=\alpha+\beta-K_{21}-K_{\mathrm{e}}, \\
& V_{\mathrm{c}}=\frac{K_{\mathrm{a}} F D}{A\left(K_{\mathrm{a}}-\alpha\right)+B\left(K_{\mathrm{a}}-\beta\right)},
\end{aligned}
$$

For calculation of $V_{\mathrm{c}}$, the value of $F$ (the absorption ratio) was assumed to be “1."

\section{RESULTS AND DISCUSSION}

To analyze the blood thiamin profiles caused by administration of TTFD, we used the change in the blood thiamin level from the baseline (7). This method can 
Table 1. Mean blood thiamin levels after a single 100-mg dose of TTFD $(n=6)$ $(5,6)$

\begin{tabular}{lcccccccc}
\hline & \multicolumn{7}{c}{ Mean blood thiamin level $(\mu \mathrm{g} /$ liter $)$} \\
\cline { 2 - 9 } & Baseline & $0.5 \mathrm{~h}$ & $1 \mathrm{~h}$ & $2 \mathrm{~h}$ & $3 \mathrm{~h}$ & $6 \mathrm{~h}$ & $24 \mathrm{~h}$ & $48 \mathrm{~h}$ \\
\hline Tablets & 42.0 & 167.8 & 322.9 & 376.0 & 321.8 & 233.5 & 103.2 & 57.2 \\
$\begin{array}{l}\text { Change from } \\
\quad \text { baseline }\end{array}$ & & 125.8 & 280.9 & 334.0 & 279.8 & 191.5 & 61.2 & 15.2 \\
$\begin{array}{l}\text { Powder } \\
\begin{array}{l}\text { Change. from } \\
\text { baseline }\end{array}\end{array}$ & 63.3 & 295.5 & 356.0 & 345.2 & 283.5 & 221.9 & 107.7 & 80.1 \\
\hline
\end{tabular}

become inaccurate if the blood thiamin level does not rise sufficiently following administration of the vitamin preparation, since the corrected values will be affected by fluctuations of the baseline level and consequently the parameters obtained by simulation may not be valid.

However, in the case of administration of $100 \mathrm{mg}$ of TTFD, the peak blood thiamin level was almost 10 times the baseline level as shown in Table 1, so that the data obtained would not be greatly influenced by fluctuations of the baseline level. The time course of the blood thiamin level over $48 \mathrm{~h}$ could therefore be simulated using a two-compartment model. After the administration of TTFD tablets, a pharmacokinetic model with a lag time was better for simulation, because absorption of thiamin was delayed until disintegration of the tablet occurred. In contrast, a model without a lag time was better in the case of TTFD powder. The simulated curves are shown in Fig. 2 (A: tablets; B: powder), and the pharmacokinetic parameters obtained by simulation are listed in Table 2 .

The pharmacokinetic parameters obtained from the studies of TTFD administration in tablet and powder form were almost identical. The relatively large distribution volume calculated was probably due to the fact that thiamin mainly localized in the erythrocytes in the blood. However, the physiological meaning of the distribution volume could not be explained by this study. The high value of the absorption rate constant is compatible with the fact that TTFD is readily absorbed via the gastrointestinal tract in contrast to the water-soluble thiamin salts.

Following the administration of $10 \mathrm{mg}$ of TTFD, the maximum increase in the blood thiamin level from the baseline was only $30-40 \mu \mathrm{g} /$ liter, while the normal baseline value was $40-50 \mu \mathrm{g} /$ liter as shown in Table 3. Therefore, the blood thiamin data obtained after the administration of TTFD were probably significantly influenced by fluctuations of the baseline level. Despite this, blood thiamin profiles for a 10-mg dose of TTFD could also be simulated reasonably using the twocompartment model. The pharmacokinetic parameters obtained are summarized in Table 4. The pharmacokinetic parameters obtained for the 10-mg dose varied somewhat between the two studies, with differences in the distribution rate constant and distribution volume being particularly large. These parameters were also 

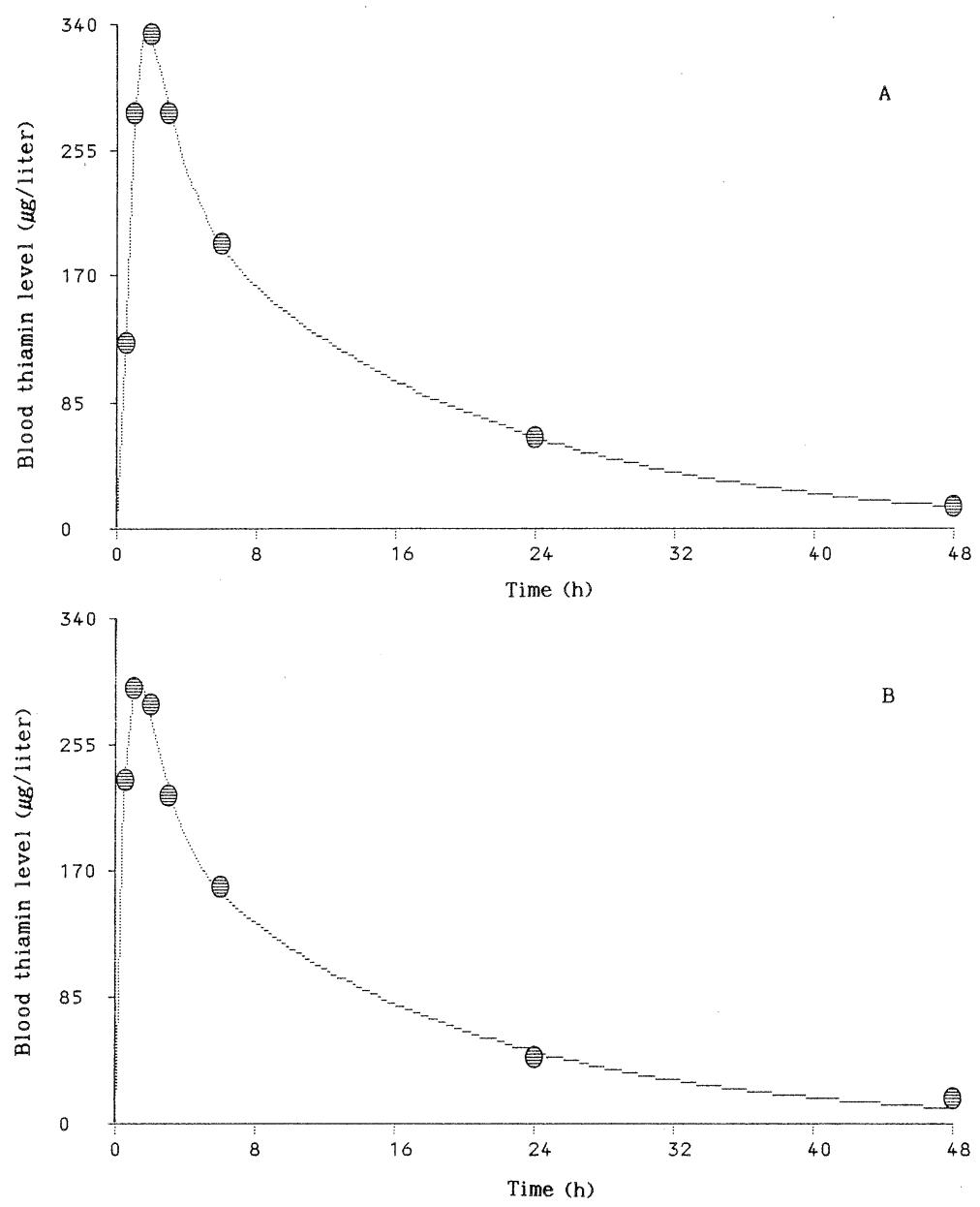

Fig. 2. Simulation curves of blood thiamin level after a single dose of $100 \mathrm{mg}$ TTFD. The plots represent the data above the baseline level. A: Tablets; B: Powder.

different from those obtained at a dose of $100 \mathrm{mg}$. Such differences were probably caused by the smaller increase in the blood thiamin level above the baseline at a dose of $10 \mathrm{mg}$, as mentioned above.

Accordingly, the pharmacokinetic parameters derived from analysis of the data for a dose of $100 \mathrm{mg}$ were next applied to the data for a 10-mg dose. After substituting the constants $A$ and $B$ with values that were $1 / 10$ of the original ones because of the dose-dependent as well as $\alpha$ and $\beta$ in Eq. (6), the blood thiamin profile for a 10-mg dose was estimated. The curve estimated and the data obtained at a dose of $10 \mathrm{mg}$ are shown in Fig. 3 (A: data for day 1 during the 1-week repeated 
Table 2. Pharmacokinetic parameters of thiamin after a 100-mg dose of TTFD.

\begin{tabular}{lcc}
\hline & TTFD tablets & Powder \\
\hline$K_{12}\left(\mathrm{~h}^{-1}\right)$ & 0.406 & 0.465 \\
$K_{21}\left(\mathrm{~h}^{-1}\right)$ & 0.375 & 0.428 \\
$K_{\mathrm{a}}\left(\mathrm{h}^{-1}\right)$ & 1.17 & 1.34 \\
$K_{\mathrm{e}}\left(\mathrm{h}^{-1}\right)$ & 0.137 & 0.158 \\
$V_{\mathrm{c}}($ liter $)$ & 163 & 181 \\
Lag time $(\mathrm{h})$ & 0.292 & - \\
\hline
\end{tabular}

Table 3. Mean blood thiamin levels following single or repeated administration of 10-mg dose of TTFD $(n=6)(4)$.

\begin{tabular}{lccccccc}
\hline & \multicolumn{7}{c}{ Mean blood thiamin level $(\mu \mathrm{g} /$ liter $)$} \\
\cline { 2 - 8 } & Baseline & $0.5 \mathrm{~h}$ & $1 \mathrm{~h}$ & $2 \mathrm{~h}$ & $3 \mathrm{~h}$ & $6 \mathrm{~h}$ & $24 \mathrm{~h}$ \\
\hline $\begin{array}{l}\text { TTFD tablets } \\
\begin{array}{c}\text { Change from } \\
\text { baseline }\end{array}\end{array}$ & 45.3 & 39.8 & 74.0 & 76.8 & 77.2 & 71.8 & 84.3 \\
$\begin{array}{l}\text { Multivitamin } \\
\begin{array}{c}\text { Change from } \\
\text { baseline }\end{array}\end{array}$ & 40.9 & 42.1 & 69.0 & 84.0 & 77.2 & 68.4 & 59.5 \\
$\begin{array}{c}\text { Multivitamin (day 1 } \\
\text { of repeated admin.) }\end{array}$ & 44.8 & 43.0 & 70.2 & 74.3 & 74.3 & 67.3 & 53.3 \\
$\begin{array}{c}\text { Change from } \\
\text { baseline }\end{array}$ & & -1.8 & 25.4 & 29.5 & 29.5 & 22.5 & 8.5 \\
$\begin{array}{c}\text { Multivitamin (day 7 } \\
\text { of repeated admin.) }\end{array}$ & 57.6 & 63.1 & 82.0 & 84.7 & 83.5 & 81.7 & 65.3 \\
$\begin{array}{c}\text { Change from } \\
\text { baseline }\end{array}$ & 5.5 & 24.4 & 27.1 & 25.9 & 24.1 & 7.7 \\
\hline
\end{tabular}

Table 4. Pharmacokinetic parameters of thiamin after a 10-mg dose of TTFD.

\begin{tabular}{lccccc}
\hline & \multicolumn{2}{c}{ Single dose of } & & \multicolumn{2}{c}{$\begin{array}{c}\text { Repeated dose of } \\
\text { multivitamin tablets }\end{array}$} \\
\cline { 2 - 3 } \cline { 5 - 6 } & $\begin{array}{c}\text { TTFD } \\
\text { tablets }\end{array}$ & $\begin{array}{c}\text { Multivitamin } \\
\text { tablets }\end{array}$ & & Day 1 & Day 7 \\
\hline$K_{12}\left(\mathrm{~h}^{-1}\right)$ & 0.856 & 0.369 & & 0.225 & 0.0003 \\
$K_{21}\left(\mathrm{~h}^{-1}\right)$ & 1.20 & 0.279 & & 2.81 & 3.12 \\
$K_{\mathrm{a}}\left(\mathrm{h}^{-1}\right)$ & 2.10 & 1.28 & & 3.05 & 3.12 \\
$K_{\mathrm{e}}\left(\mathrm{h}^{-1}\right)$ & 0.038 & 0.040 & & 0.065 & 0.055 \\
$V_{\mathrm{c}}($ liter $)$ & 185 & 148 & & 287 & 334 \\
Lag time $(\mathrm{h})$ & 0.500 & 0.500 & & 0.517 & 0.435 \\
\hline
\end{tabular}

dose study; B: data for last day during the 1-week repeated dose study; estimated curves are the same).

As shown in Fig. 3, the blood thiamin profile estimated using 1/10 values for 

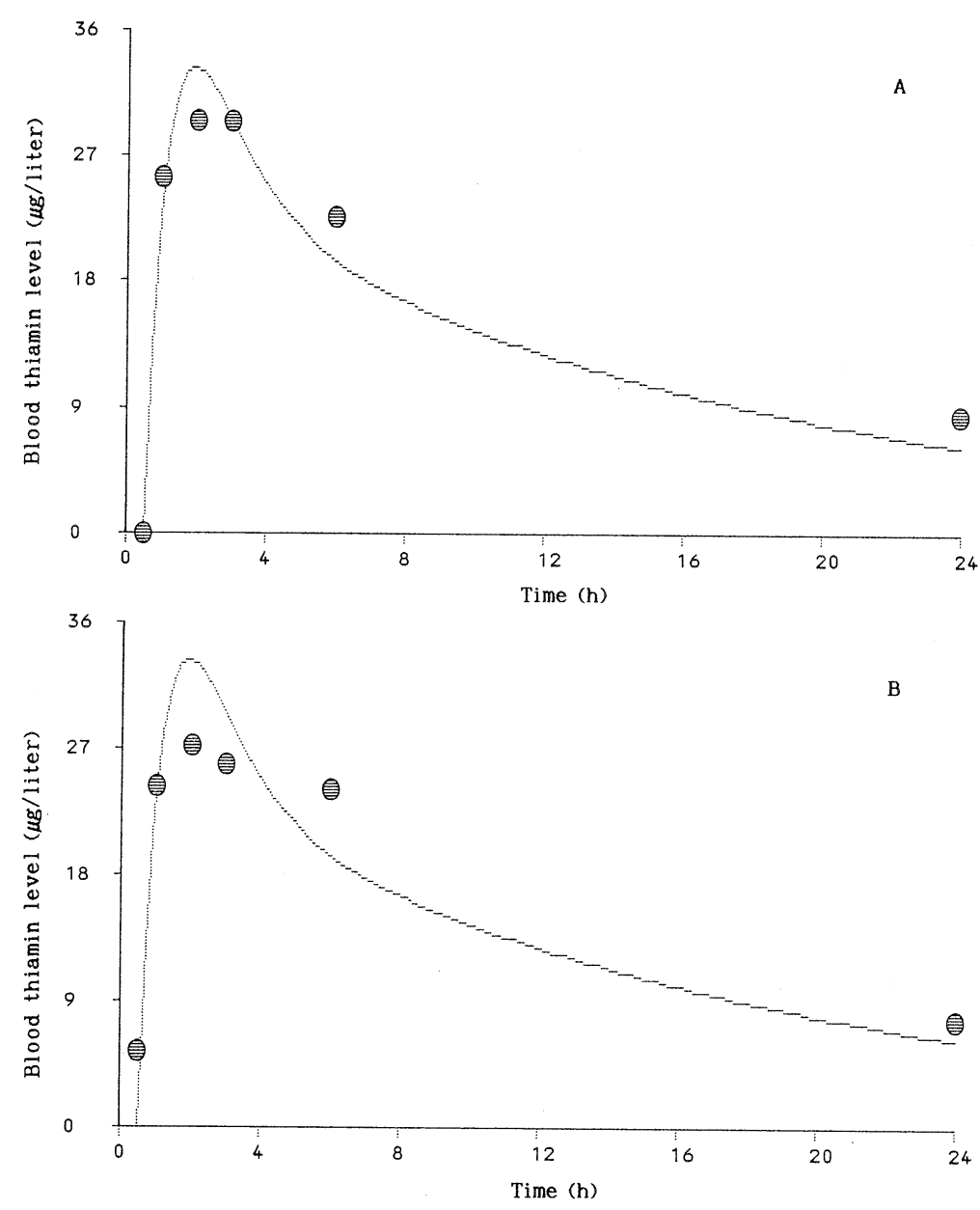

Fig. 3. Estimated blood thiamin level profiles for a 10-mg dose of TTFD using pharmacokinetic parameters obtained at a dose of $100 \mathrm{mg}$. A: The points represent the data above the baseline level on day 1 during the 1-week repeated dose study. B: The points represent the data above the baseline level on day 7 during the 1-week repeated dose study.

the constants $A$ and $B$ obtained at a dose of $100 \mathrm{mg}$ were in good agreement with the data of the 10-mg studies. This result suggests that the pharmacokinetic parameters obtained from the 100-mg studies were more reliable than those from 10-mg studies.

The fact that the pharmacokinetic parameters obtained from the 100-mg studies on the tablet and powder forms of TTFD were almost identical, while those obtained from the four 10-mg studies were different, also suggests that the former 
set of parameters were more reliable. The rate of the oral absorption generally decreases with an increase in the dose. The fact that the parameters of the 10-mg dose were equal to the ones of $100-\mathrm{mg}$ dose means that the parameters are not dose-dependent and therefore the absorption ratio is equivalent between two doses.

Aso et al. (3) administered $30 \mathrm{mg}$ of TTFD labeled with ${ }^{35} \mathrm{~S}$ at the $\mathrm{VB}_{1}$ moiety together with $150 \mathrm{mg}$ of unlabeled compound to humans, and found that it was completely absorbed as thiamin. Since even $180 \mathrm{mg}$ of TTFD was completely absorbed, 10 or $100 \mathrm{mg}$ of TTFD would be completely absorbed from the gastrointestinal tract. Therefore, it was felt to be appropriate to assume that the absorption ratio $(F)$ was unity when the simulation was carried out.

\section{REFERENCES}

1) Menke, G. (1990): Wird Vitamin $B_{1}$ nach orale Gabe resorbiert? Dtsch. Apoth. Ztg., 130, 491-494.

2) Baker, H., Thomson, A. D., Frank, O., and Leevy, C. M. (1974): Absorption and passage of fat- and water-soluble thiamin derivatives into erythrocytes and cerebrospinal fluid of man. Am. J. Clin. Nutr., 27, 676-680.

3) Aso, K., Tanabe, Y., Takeuchi, K., Kobayashi, T., and Shiomi, T. (1965): On the absorption and excretion of thiamine derivatives 8 . The absorption and excretion of thiamine tetrahydrofurfuryl disulfide $-{ }^{35} \mathrm{~S}$ (inner). Vitamin (Jpn.), 32, 387-394.

4) Itokawa, Y., Takeuchi, T., Nishino, K., Matsuoka, M., and Otsuka, H. (1989): Blood levels and urinary excretion of vitamin $\mathrm{B}_{1}$ and vitamin $\mathrm{B}_{2}$ following oral administration of vitamin preparations. Vitamin (Jpn.), 63, 503-511.

5) Itokawa, Y., Nishino, K., and Igarashi, S. (1992): Evaluation of thiamin derivatives: Human bioavailability, uptake by human blood cells, and conversion to thiamin by rat liver homogenate. Vitamin (Jpn.), 66, 35-42.

6) Itokawa, Y., Takeuchi, T., Nishino, K., Matsuoka, M., and Otsuka, H. (1992): Blood levels and urinary excretion of vitamin $B_{1}$ following oral administration of thiamin tetrahydrofurfuryl disulfide powder. Vitamin (Jpn.), 66, 477.

7) Kitamori, N. (1992): Some considerations on the pharmacokinetic analysis of vitamins, in The Proceedings of the 1st International Congress on "Vitamins and Biofactors in Life Science," pp. 442-445.

8) Yamaoka, K., and Tanigawara, Y. (1983): Introduction to Pharmacokinetics Using a Microcomputer, Nankodo, Inc., Tokyo, pp. 33-50. 